typeset using JPSJ.sty $<$ ver.1.0b $>$

\title{
Resonant X-Ray Scattering from the Quadrupolar Ordering Phase of $\mathrm{CeB}_{6}$
}

\author{
Tatsuya NAgAot and Jun-ichi IGARASHI \\ Faculty of Engineering, Gunma University, Kiryu, Gunma 376-8515
}

(Received

)

\begin{abstract}
We theoretically investigate the origin of the resonant x-ray scattering (RXS) signal near the Ce $L_{\text {III }}$ absorption edge in the quadrupolar ordering phase of $\mathrm{CeB}_{6}$, considering the intersite interaction between the $\Gamma_{8}$ states in the initial state. The anisotropic charge distribution of the $4 f$ states modulates the $5 d$ states through the intra-atomic Coulomb interaction and thereby generates a large RXS superlattice intensity. The temperature and magnetic field dependence indicates that the induced dipolar and octupolar orders have little influence on the RXS spectra, in good agreement with the recent experiment.
\end{abstract}

KEYWORDS: resonant x-ray scattering, $\mathrm{CeB}_{6}$, orbital ordering, $\mathrm{Ce} L_{\mathrm{III}}$ absorption edge

Resonant x-ray scattering (RXS) has attracted much interest as a useful tool to investigate the orbital order, since the resonant enhancement on the superlattice Bragg spots for the orbital order was observed near the Mn $K$ edge in $\mathrm{LaMnO}_{3}$. .t Although similar experiments have already been carried out on several materials, the origin of the RXS signal is still controversial. The RXS is described by a second order process that photons are virtually absorbed by exciting a core electron to unoccupied states and then emitted by recombining the excited electron with the core hole. Therefore, the $4 p$ states are involved near the $K$ edge for $\mathrm{LaMnO}_{3}$ in the dipolar process. In the early stage, the Coulomb interaction between the $3 d$ and $4 p$ states was considered as the origin, 2, 3) but subseguent studies based on the band structure calculations, 6. 6) have revealed that the Coulomb effect is two order of magnitude smaller than the effect of the Jahn-Teller distortion, indicating that the $4 p$ states are sensitive to electronic structure of neighboring sites.

Recently the RXS experiment was carried out near the Ce $L_{\mathrm{III}}$ absprption edge in the quadrupolar ordering phase of $\mathrm{CeB}_{6}$. The lattice distortion is not observed because of much weaker coupling between lattice and well-localized $4 f$ states. Also, the $5 d$ states are considered to be more localized than the $4 p$ states in $\mathrm{LaMnO}_{3}$. Therefore, the mechanism of the RXS in $\mathrm{CeB}_{6}$ is expected to be different from that in $\mathrm{LaMnO}_{3}$. The purpose of this paper is to make clear the origin and to discuss the implication of the RXS spectra in comparison with the experiment, by considering the intersite interaction between the $\Gamma_{8}$ states.

In $\mathrm{CeB}_{6}$, each $\mathrm{Ce}$ atom is considered to be in the $f^{1}$ configuration. Within the $J=5 / 2$ subspace, the $\Gamma_{8}$ quartet states have lower energy than the $\Gamma_{7}$ doublet under the cubic crystal field. The $\Gamma_{8}-\Gamma_{7}$ excitation energy is $\sim 540 \mathrm{~K}$. With decreasing temperatures the antiferro quadrupole (AFQ) order appears at $T_{Q}=3.3 \mathrm{~K}$ with an ordering wave vector $\mathrm{Q}=(1 / 2,1 / 2,1 / 2)$. Antiferromagnetic phases exist at even lower temperatures, but will not be considered in this paper. These phase transitions originate from the intersite interaction, which lifts the degeneracy of the $\Gamma_{8}$ states.

The intersite interaction was derived on the basis of a RKKY interaction by Ohkawa. 10 Recently, Shiina et al.11 2.213) extended his model by fully taking account of the symmetry of the interaction as well as the order parameters. Thereby, they solved a longstanding controversy between the neutron diffraction 144 and NMR 15 in the context of the induced order parameters under the external magnetic field. Following their study, we use in the initial state the Hamiltonian $\tilde{H}$ of the so called extended Ohkawa model::13)

$$
\begin{aligned}
\tilde{H}= & D \sum_{\langle i, j\rangle}\left[(1+\delta) \boldsymbol{\mu}_{i} \cdot \boldsymbol{\mu}_{j}+\tau_{i}^{y} \tau_{j}^{y}+\epsilon \boldsymbol{\sigma}_{i} \cdot \boldsymbol{\sigma}_{j}\right. \\
& \left.+\frac{1+\epsilon}{2}\left(\boldsymbol{\tau}_{i}^{\prime} \cdot \boldsymbol{\tau}_{j}^{\prime}+\boldsymbol{\eta}_{i} \cdot \boldsymbol{\eta}_{j}+\boldsymbol{\zeta}_{i} \cdot \boldsymbol{\zeta}_{j}\right)\right] \\
& -2 \mu_{\mathrm{B}} \sum_{i}\left(\boldsymbol{\sigma}_{i}+\frac{4}{7} \boldsymbol{\eta}_{i}\right) \cdot \mathbf{H},
\end{aligned}
$$

where $\boldsymbol{\mu}, \boldsymbol{\tau}^{\prime}, \boldsymbol{\eta}, \boldsymbol{\zeta}$ acting on the $\Gamma_{8}$ states are explicitly defined in ref. 13 . Since the RXS spectra are not sensitive to the parameter values of $\delta$ and $\epsilon$, we simply assume $\delta=0.2$ and $\epsilon=1$. This choice is knoyn to give a reasonable description for the AFQ phase.11, 12, 13 Assuming $D=1.83 \mathrm{~K}$, we get the transition temperature $T_{Q}=3.3 \mathrm{~K}$ for the AFQ phase with no external magnetic field in the mean field approximation.

Near the Ce $L_{\text {III }}$ absorption edge, the $2 p$ core electron is virtually excited to the $5 d$ states and is recombined with the core hole in the dipolar process. Since the $2 p$ states are well localized around Ce sites, the scattering tensor can be approximated by a sum of the contributions from each site of the created core hole. The corre- 
sponding cross section is given by

$$
\left.\frac{d \sigma}{d \Omega}\right|_{\mu \rightarrow \mu^{\prime}} \propto\left|\sum_{\alpha \alpha^{\prime}} P_{\alpha}^{\prime \mu^{\prime}} M_{\alpha \alpha^{\prime}}(\mathbf{G}, \omega) P_{\alpha^{\prime}}^{\mu}\right|^{2},
$$

with

$$
\begin{aligned}
& M_{\alpha \alpha^{\prime}}(\mathbf{G}, \omega)=\frac{1}{\sqrt{N}} \sum_{j} \sum_{n, \Lambda} p_{n}(j) \\
& \times \frac{\left\langle\psi_{n}(j)\left|x_{\alpha}(j)\right| \Lambda\right\rangle\left\langle\Lambda\left|x_{\alpha^{\prime}}(j)\right| \psi_{n}(j)\right\rangle}{\hbar \omega-\left(E_{\Lambda}-E_{n}(j)\right)+i \Gamma} \exp \left(i \mathbf{G} \cdot \mathbf{r}_{j}\right) .
\end{aligned}
$$

Here $\mathbf{G}$ is the scattering vector, and $\omega$ is the frequency of photon. The $j$ runs over Ce sites. The cross section becomes order $N$, the number of Ce sites. The dipole operators $x_{\alpha}(j)$ 's are defined as $x_{1}(j)=x, x_{2}(j)=y$, and $x_{3}(j)=z$ in the coordinate frame fixed to the crystal axes with the origin located at the center of site $j$. The energy eigenvalues are defined as $E_{n}(j)(n=1 \sim 4)$ and the eigenfunctions as $\left|\psi_{n}(j)\right\rangle$ at site $j$ in the mean field approximation. We take thermal average on these states with probability $p_{n}(j)$. State $|\Lambda\rangle$ represents the intermediate state with energy $E_{\Lambda}$. The life-time broadening width $\Gamma$ of the core hole is assumed to be $2 \mathrm{eV}$. We study only the situation of $\mathbf{G}=(1 / 2,1 / 2,1 / 2)$ in the following. For this $\mathbf{G}$ value in the conventional scattering geometry 1) the geometrical factors $P^{\mu}$ for incident photons and $P^{\prime \mu^{\prime}}$ for scattered photons are given by 16$)\left(P^{\sigma}\right)_{1}=$ $\left(P^{\prime \sigma^{\prime}}\right)_{1}=(\cos \beta \cos \psi+\sin \psi) / \sqrt{2},\left(P^{\sigma}\right)_{2}=\left(P^{\prime \sigma^{\prime}}\right)_{2}=$ $(\cos \beta \cos \psi-\sin \psi) / \sqrt{2},\left(P^{\sigma}\right)_{3}=\left(P^{\prime \sigma^{\prime}}\right)_{3}=-\sin \beta \cos \psi$, $\left(P^{\prime \pi^{\prime}}\right)_{1}=[-\sin \theta(\cos \beta \sin \psi-\cos \psi)+\cos \theta \sin \beta] / \sqrt{2}$, $\left(P^{\prime \pi^{\prime}}\right)_{2}=[-\sin \theta(\cos \beta \sin \psi+\cos \psi)+\cos \theta \sin \beta] / \sqrt{2}$, $\left(P^{\prime \pi^{\prime}}\right)_{3}=[\sin \theta \sin \beta \sin \psi+\cos \theta \cos \beta]$, with $\theta$ being the Bragg angle and $\beta=\arccos 1 / \sqrt{3}$. The azimuthal angle $\psi$ is defined such that the crystal axis $(1,-1,0)$ lies on the scattering plane at $\psi=0$.

In the intermediate state, the $5 d$ states of Ce are involved. From the band calculation for $\mathrm{LaB}_{6}, 17$ it is known that the $5 d$ states of La hybridize with B $2 p$ states, forming energy bands with their width $\sim 5 \mathrm{eV}$. The $5 d$ states in $\mathrm{CeB}_{6}$ are considered to be similar to those in $\mathrm{LaB}_{6}$, except for the relative position to the $4 f$ levels. Note that the $5 d$ states are more localized than the $4 p$ states in $\mathrm{LaMnO}_{3}$, since the latter band width is as large as $\sim 20 \mathrm{eV}$. Therefore, the dominant contribution to the RXS spectra comes from the local process which can be expressed in terms of the local Green's function constructed by the $5 d$ density of states (DOS). We roughly simulate it by a simple rectangular shape with the width $5(2) \mathrm{eV}$ and the edge located $2.5(5.5) \mathrm{eV}$ above the $4 f$ levels, for the $t_{2 g}\left(e_{g}\right)$ symmetry. The $5 d$ band is slightly filled by electrons, which mediate the intersite interaction mentioned above.9.10,13. In the present calculation, however, we simply assume the vacant $5 d$ band in the initial state, since the actual filling modifies little the RXS spectra. Thus, the local Green's function for the $5 d$ states is defined by

$$
G_{m^{d}, m^{\prime} d}^{5 d}(\omega)=\delta_{m^{d} m^{\prime d}} \int \frac{\rho_{m^{d}}^{5 d}(\epsilon)}{\omega-\epsilon+i \delta} \mathrm{d} \epsilon,
$$

where $\rho_{m^{d}}^{5 d}(\epsilon)$ is the DOS mentioned above with $m^{d}$ in- dicating either $t_{2 g}$ or $e_{g}$ states.

The intermediate state at the site of core hole are specified by $m^{d} s^{d}\left(s^{d}\right.$ : spin) of the $5 d$ electron, $m^{f} s^{f}\left(m^{f}=\right.$ $-3, \ldots, 3)$ of the $4 f$ electron, and $m^{p} s^{p}\left(m^{p}=-1,0,1\right)$ of the $2 p$ hole. Then the resolvent in the space of these states is given by

$$
\begin{aligned}
& \left(\frac{1}{\omega-H_{\mathrm{int}}+i \delta}\right)_{m^{d} s^{d} m^{f} s^{f} m^{p} s^{p} ; m^{\prime d} s^{\prime d} m^{\prime f} s^{\prime f} m^{\prime p} s^{\prime p}} \\
= & {\left[G_{m^{d}, m^{\prime d}}^{5 d}\left(\omega+i \Gamma-\epsilon_{f}-\epsilon_{p}\right)^{-1}\right.} \\
& \left.-V_{m^{d} s^{d} m^{f} s^{f} m^{p} s^{p} ; m^{\prime d} s^{\prime d} m^{\prime f} s^{\prime f} m^{\prime p} s^{\prime p}}\right]^{-1},
\end{aligned}
$$

where $H_{\text {int }}$ represents the Hamiltonian in the intermediate state. The $\epsilon_{f}$ and $\epsilon_{p}$ represent the average energy of the $4 f$ electron and $2 p$ hole. The interaction $V$ contains the spin-orbit interaction for each particles, and the intra-atomic Coulomb interaction between them. The parameter values for them are evaluated in the $2 p^{5} 4 f^{1} 5 d^{1}$ configuration of the $\mathrm{Ce}^{3+}$ atom within the Hartree-Fock approximation 18) It is known that the Slater integrals $F^{0}(5 d, 4 f), F^{0}(2 p, 4 f)$ and $F^{0}(2 p, 5 d)$ are well screened while the other integrals are not much even in solids. We assume relatively small values, $4 \mathrm{eV}, 12 \mathrm{eV}$, and $7 \mathrm{eV}$ for $F^{0}(5 d, 4 f), F^{0}(2 p, 4 f)$ and $F^{0}(2 p, 5 d)$, respectively, while we use the atomic values multiplied by a factor 0.8 for other quantities. Note that the anisotropic parts $F^{2}(5 d, 4 f), F^{4}(5 d, 4 f), G^{1}(5 d, 4 f), G^{3}(5 d, 4 f), G^{5}(5 d, 4 f)$ in the Coulomb interaction between the $5 \mathrm{~d}$ and $4 \mathrm{f}$ electrons, not the isotropic part $F^{0}(5 d, 4 f)$, are relevant to giving rise to the RXS intensity. The crystal field energy, which is important for selecting the $\Gamma_{8}$ states as the initial state, is negligibly small in the calculation of the intermediate state. The right hand side of eq. (5) is a matrix with dimensions $840 \times 840$, which we numerically invert. This resolvent is the same at all sites of core hole, so that the scattering amplitudes become different at different sublattices of the AFQ order only after multiplying the matrix elements of the dipole operators between the initial and the intermediate state (see eq. (3)).

Now we discuss the calculated results. We have three types of possible ordered phase for $H=0$, in which one of the staggered quadrupole moments, $\left\langle O_{x y}\right\rangle\left(\equiv 4\left\langle\tau_{y} \sigma_{z}\right\rangle\right)$, $\left\langle O_{y z}\right\rangle\left(\equiv 4\left\langle\tau_{y} \sigma_{x}\right\rangle\right)$, and $\left\langle O_{z x}\right\rangle\left(\equiv 4\left\langle\tau_{y} \sigma_{y}\right\rangle\right)$, is finite. Here $\langle X\rangle$ indicates the thermal average of operator $X$. We simply call them as the $O_{x y}, O_{y z}$, and $O_{z x}$ phases. Assuming three domains with equal relative volumes, we average the RXS intensities over the domains. Figure 1 shows the RXS spectrum as a function of photon energy, at $T=2.7 \mathrm{~K}$ and $\psi=0$. We obtain a single peak structure in agreement with the experiment. \&) We adjusted the core hole energy $\epsilon_{p}$ such that the peak is located at the experimental position $\hbar \omega=5722 \mathrm{eV}$. The $\omega$-dependence is the same in the three domains. We roughly estimate that the peakintensity is less than $10^{-2}$ or $10^{-3}$ of that for $\mathrm{LaMnO}_{3}$, 4 , The contribution of the quadrupolar process is included in the curve.19. Although it is expected to give a peak in the pre-edge region, it is too small to be seen. This contrasts with the RXS spectra from the $\mathrm{AFQ}$ phase of $\mathrm{DyB}_{2} \mathrm{C}_{2}$, where a pre-edge 
peak is conspicuous.20 21 The core hole potential cannot become a main origin for the RXS spectra, since it works the same way at all sites. This point is clarified by comparing the spectra with those calculated by turning off the potential. The latter also consists of a single peak located at the position about $19 \mathrm{eV}$ higher than the former. In the figure, the spectrum is shifted such that the peak is located at the same position; the spectral shape and intensity are similar except for the energy shift. This behavior is quite different from the absorption spectra, which are known to be considerably enhanced by the core hole potential.

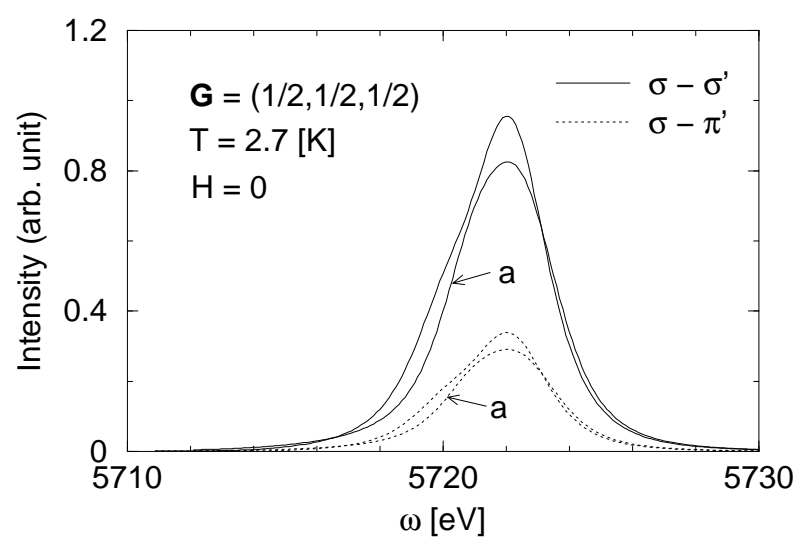

Fig. 1. RXS spectra as a function of photon energy for $\mathbf{G}=$ $(1 / 2,1 / 2,1 / 2) . H=0, T=2.7 \mathrm{~K}$, and $\psi=0$. The contributions from three domains are averaged. The solid and dotted lines represent the intensities for the $\sigma \rightarrow \sigma^{\prime}$ channel and the $\sigma \rightarrow$ $\pi^{\prime}$ channel, respectively. Curves with letter "a" represent the spectra calculated by turning off the core hole potential.

We obtain the scattering amplitudes $M(\mathbf{G}, \omega)$ in simple forms,

$$
\left(\begin{array}{lll}
0 & a & 0 \\
a & 0 & 0 \\
0 & 0 & 0
\end{array}\right),\left(\begin{array}{lll}
0 & 0 & 0 \\
0 & 0 & a \\
0 & a & 0
\end{array}\right),\left(\begin{array}{lll}
0 & 0 & a \\
0 & 0 & 0 \\
a & 0 & 0
\end{array}\right)
$$

in the $O_{x y}, O_{y z}$, and $O_{z x}$ phases, respectively. Therefore, the principal axes, for example in the $O_{x y}$ phase, are given by rotating the $x, y$ axes with $\pi / 4$ around the $z$ axis. Note that the eigenstates with the eigenvalue 1 (1) for the operator $O_{x y}$ has a larger charge distribution along the $(1,-1,0)((1,1,0))$ direction. The principal axes are consistent with the anisotropic charge density of the $4 f$ states in the AFQ phase. This contrasts with the situation of $\mathrm{LaMnO}_{3}$, where the neighboring oxygen potentials aremore important through the Jahn-Teller distortion. 460

Figure 2 shows the azimuthal angle dependence of the intensity of the peak at $\hbar \omega=5722 \mathrm{eV}$. The contributions from the three domains are separately shown. The dependence for the $\sigma \rightarrow \sigma^{\prime}$ channel is quite different from that for the $\sigma \rightarrow \pi^{\prime}$ channel. The curves for the $O_{x y}$, $O_{y z}$ and $O_{z x}$ phases can be transformed into those for the $O_{y z}, O_{z x}$, and $O_{x y}$ phases, respectively, by shifting $\psi$ with $2 \pi / 3$. This threefold symmetry around the $(1,1,1)$ direction perfectly matches the relation between the or- der parameters of the three domains.

Under the external magnetic field, the staggered octupole moment and the staggered dipole moment are induced in addition to the staggered quadrupole moment. To make clear the effects of such induced moments on the RXS spectra, we calculate the RXS intensity of the peak at $\hbar \omega=5722 \mathrm{eV}$ as a function of $H$. The temperature is set rather low, $T=1.37 \mathrm{~K}$, so that the quadrupole order parameter is sufficiently developed for all values of $H$ (the possible antiferromagnetic phase is disregarded). Figure 3 shows the calculated results in comparison with the order parameters. For $H \|(001)$, the primary order parameter is the staggered quadrupole moment of $\left\langle O_{x y}\right\rangle$. The staggered octupole moment $\left\langle T_{x y z}\right\rangle\left(\equiv 2\left\langle\tau^{y}\right\rangle\right)$ is induced with increasing $H$, but the staggered dipole moment is not. As shown in the left panel, the RXS intensity changes little with changing $H$, indicating that the induced octupole moment is irrelevant to the RXS spectra. For $H \|(110)$, the primary order parameter is the staggered quadrupole moment $\left\langle O_{y z}+O_{z x}\right\rangle / \sqrt{2}$, and the staggered dipole moment $\left\langle J_{z}\right\rangle\left(\equiv\left\langle\frac{14}{11} \sigma^{z}+\frac{16}{11} \tau^{z} \sigma^{z}\right\rangle\right)$ is induced in addition to the induced octupole moment with increasing $H$. As shown in the right panel, the RXS intensity changes little with increasing $\mathrm{H}$, indicating that the induced dipole moment is also irrelevant to the RXS spectra. This marks the difference between the RXS and the neutron scattering experiment the latter of which detects the induced dipole moment.14
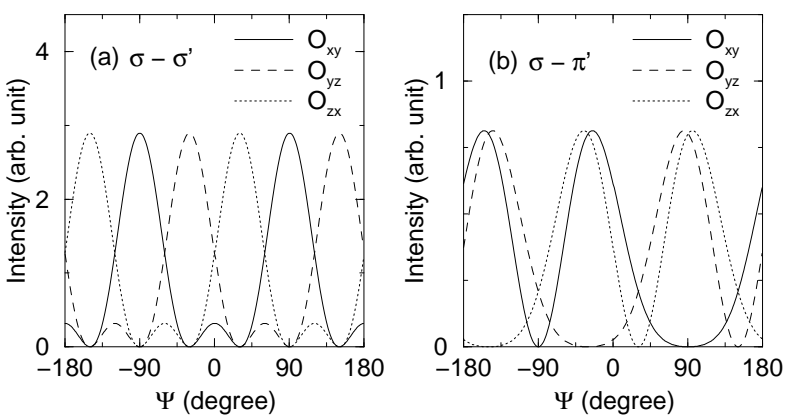

Fig. 2. Azimuthal angle dependence of the RXS intensity of the peak at $\hbar \omega=5722 \mathrm{eV}$, for (a) the $\sigma \rightarrow \sigma^{\prime}$ channel and (b) the $\sigma \rightarrow \pi^{\prime}$ channel. $T=2.7 \mathrm{~K}$ and $H=0$. The solid, broken, and dotted lines represent the contributions from the $O_{x y}, O_{y z}$, and $O_{z x}$ phases, respectively.

Figure 4 shows the temperature dependence of the RXS intensity of the peak at $\hbar \omega=5722 \mathrm{eV}$, for $H=0$ and for several values of $H \|(1,1,-2)$. The inset shows the staggered order parameter $\left(\left\langle O_{x y}\right\rangle\right.$ in the $O_{x y}$ phase) for $H=0$ and its square, calculated within the mean field approximation. The temperature dependence of the spectra for $H=0$ looks similar to that of the square of the order parameter. The dependence changes little with changing yalues of $H$, in agreement with the recent experiment.

In summary, we have calculated the RXS spectra near the Ce $L_{\mathrm{III}}$ absorption edge, considering the intersite in- 


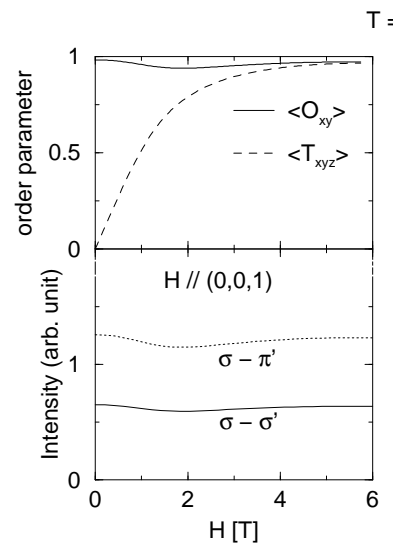

$\mathrm{T}=1.37[\mathrm{~K}]$

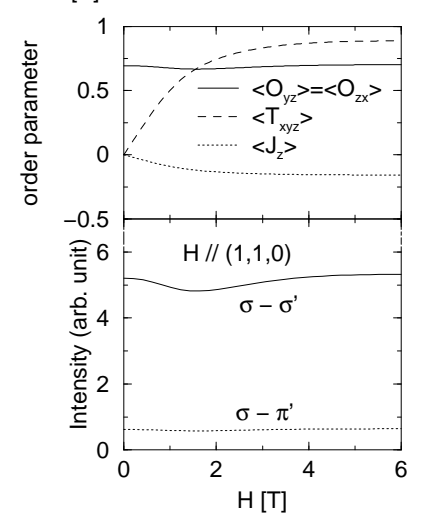

Fig. 3. RXS intensity of the peak at $\hbar \omega=5722 \mathrm{eV}$ as a function of $H$. Left panel: $H \|(001)$; Right panel: $H \|(110)$. $T=1.37$ $\mathrm{K}$ and $\psi=0$. The solid and dotted lines represent the intensities for the $\sigma \rightarrow \sigma^{\prime}$ channel and the $\sigma \rightarrow \pi^{\prime}$ channel, respectively. Upper panels show the staggered quadrupole moments, $\left\langle O_{x y}\right\rangle$, $\left\langle O_{y z}\right\rangle,\left\langle O_{z x}\right\rangle$, the staggered dipole moments $\left\langle J_{z}\right\rangle$, and the staggered octupole moment $\left\langle T_{x y z}\right\rangle$.

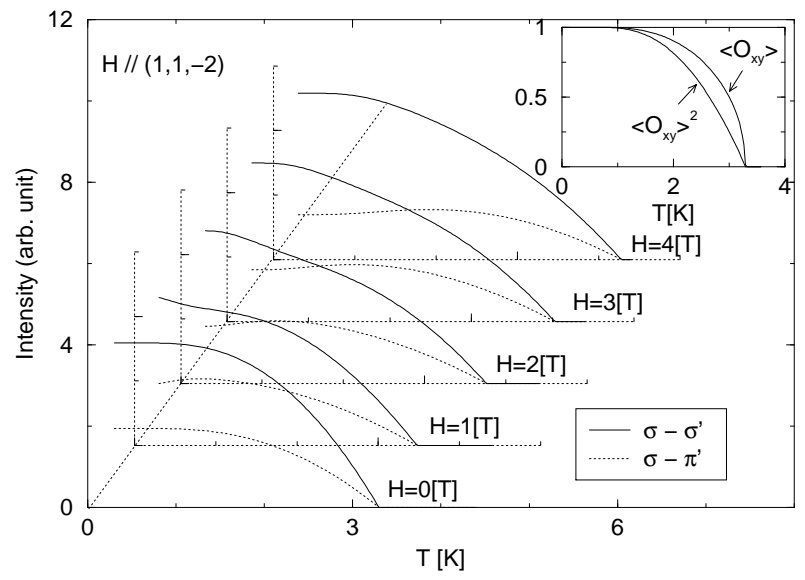

Fig. 4. Temperature dependence of the RXS intensity of the peak at $\hbar \omega=5722 \mathrm{eV}$, for $H=0$ and for finite $H \|(1,1,-2)$. The curve for $H=0$ is not the limit of $H \rightarrow 0$ but the domain average. Inset: the staggered quadrupole moment and its square for $H=0$.

teraction between the $\Gamma_{8}$ states in the initial state. We have demonstrated that the $4 f$ charge distribution modulates the $5 d$ states through the intra-atomic Coulomb interaction and thereby generates a large RXS superlattice intensity. This mechanism is different from that in $\mathrm{LaMnO}_{3}$, where the oxygen potentials at neighboring sites are much important. We have evaluated the temperature and magnetic field dependence of the RXS intensity, in good agreement with the recent experiment. The magnetic field dependence indicates that the induced dipolar and octupolar orders have little influence on the RXS intensity. Thus, it can be concluded that the RXS spectrum in $\mathrm{CeB}_{6}$ is a direct reflection of the AFQ order, in contrast with the neutron diffraction and NMR experiments. We have used a simplified model for the $5 \mathrm{~d}$ bands. A change in the $5 \mathrm{~d}$ DOS shape and the filling actually modifies the spectral intensity, but does not alter the qualitative features discussed in this paper, such as a single-peak shape, the temperature and the magnetic field dependences. A quantitative study using a more realistic model is left for future.

We would like to thank Y. Murakami, H. Nakao and H. Shiba for valuable discussion. This work was partially supported by a Grant-in-Aid for Scientific Research from the Ministry of Education, Science, Sports and Culture, Japan.

[1] Y. Murakami, J. P. Hill, D. Gibbs, M. Blume, I. Koyama, M. Tanaka, H. Kawata, T. Arima, Y. Tokura, K. Hirota and Y. Endoh: Phys. Rev. Lett. 81 (1998) 582.

[2] S. Ishihara and S. Maekawa: Phys. Rev. Lett. 80 (1998) 3799.

[3] S. Ishihara and S. Maekawa: Phys. Rev. B 58 (1998) 13449.

[4] I. S. Elfimov, V. I. Anisimov and G. Sawatzky: Phys. Rev. Lett. 82 (1999) 4264.

[5] M. Benfatto, Y. Joly and C. R. Natoli: Phys. Rev. Lett. 83 (1999) 636.

[6] M. Takahashi, J. Igarashi and P. Fulde: J. Phys. Soc. Jpn. 68 (1999) 2530.

[7] M. Takahashi, J. Igarashi and P. Fulde: J. Phys. Soc. Jpn. 69 (2000) 1614.

[8] H. Nakao, K. Magishi, Y. Murakami, K. Koyama, K. Hirota, Y. Endoh and S. Kunii: J. Phys. Soc. Jpn. 70 (2001) 1857.

[9] F. J. Ohkawa: J. Phys. Soc. Jpn. 52 (1983) 3897.

[10] F. J. Ohkawa: J. Phys. Soc. Jpn. 54 (1985) 3909.

[11] R. Shiina, H. Shiba, and P. Thalmeier: J. Phys. Soc. Jpn. 66 (1997) 1741

[12] O. Sakai, R. Shiina, H. Shiba, and P. Thalmeier: J. Phys. Soc. Jpn. 66 (1997) 3005.

[13] H. Shiba, O. Sakai, and R. Shiina: J. Phys. Soc. Jpn. 68 (1999) 1988.

[14] J. M. Effantin, J. Rossat-Mignod, P. Burlet, H. Bartholin, S. Kunii, and T. Kasuya: J. Mag. Magn. Mater. 47\& 48 (1985) 145.

[15] M. Takigawa, H. Yasuoka, T. Tanaka, and Y. Ishizawa: J. Phys. Soc. Jpn. 52 (1983) 3967.

[16] J. Igarashi and M. Takahashi: Phys. Rev. B 63 (2001) 184430.

[17] H. Harima, O. Sakai, T. Kasuya, and A. Yanase: Solid State Commun. 68 (1988) 603.

[18] R. Cowan: The Theory of Atomic Structure and Spectra (University of California Press, Berkeley, 1981).

[19] Details of the quadrupole process will be discussed elsewhere.

[20] K. Hirota, N. Oumi, T. Matsumura, H. Nakao, Y. Wakabayashi, Y. Murakami, and Y. Endoh: Phys. Rev. Lett. 84 (2000) 2706 .

[21] Y. Tanaka, T. Inami, T. Nakamura, H. Yamauchi, H. Onodera, K. Ohyama, and Y. Yamaguchi: J. Phys. Condens. Matter 11 (1999) L505. 